\title{
Classification of Partial Discharge Detection Technique in High Voltage Power Component: A Review
}

\author{
M. N. K. H. Rohani ${ }^{1}$,A. A. H. Zaidi ${ }^{2}$, A. Sakanovic, C. L. Wooi ${ }^{1}$, A. S. Rosmi ${ }^{1}$, \\ M. Isa ${ }^{1}$, B. Ismail ${ }^{1}$
}

${ }^{1}$ School of Electrical System Engineering, Pauh Putra Campus, Universiti Malaysia Perlis, 02600 Arau, Perlis, MALAYSIA

${ }^{2}$ Faculty of Engineering Technology,

Universiti Malaysia Perlis, UniCITY Alam Campus, Sungai Chuchuh, 02100 Padang Besar, Perlis, MALAYSIA

*Corresponding Author

DOI: https://doi.org/10.30880/ijie.2019.11.04.027

Received 8 August 2019; Accepted 26 August 2019; Available online 31 August 2019

\begin{abstract}
In this study, a review on categorization of partial discharge (PD) detection technique is presented. The PD detection is important in order to monitor and diagnosis the insulation health on high voltage (HV) power component. PD at high voltage transformer occurs due to the unscheduled maintenance, aging of equipment, breakdown of insulation, gas bubbles in insulation liquid, manufacturing error and etc. In order to maintain constant and high as possible of the transformer performance, it is essential to control, detect and measure the PD phenomena. There are many methods have been developed based on point of observation; optical, chemical, acoustic and electrical. However, the different methods have their own advantages and disadvantages.
\end{abstract}

Keywords: Partial Discharge, High Voltage Power Component, Optical Detection, Acoustic Detection, Chemical Detection, Electrical Detection.

\section{Introduction}

In order to obtain reliable and efficient operation of power equipment such as transformer, rotating machines, circuit breakers, current and potential transformers, online monitoring of power equipment is from big importance what seeks for new ideas in this field due to rapid growth of power sector [1]-[3]. In order to have reliable energy and power systems, knowing status and conditions of electrical equipment and its insulation is essential. One of parameters that can help in obtaining information related to conditions of insulation and equipment is PD. As well PD can give information about which insulating material is the best and can be used for equipment, device and system testing [4]. Studies has shown that main cause of failure of power equipment is degradation of insulation due to constant mechanical, electrical and thermal stress in long service life [5].

PD is electrical discharge that partially bridges the isolation between conductors, as result of electrical stress in or on the surface of insulation [6], [7]. PD occurs when locations voltage breakdown is smaller than localized electric field, with presence of void, crack, foreign material, or structural changes. Once occurred PD appears as localized release of energy causing structural changes in material, electromagnetic effects, heat and noise. Appearance of PD is in form of pulses of period less than one second with a quick transfer of charge in place of occurrence. Once produced PD emits electric impulse with high frequency that spreads through the surrounding and media. Short time of PD will not produce much defects, but through longer constant appearing time it can have dangerous effect beginning with 
degradation of insulation till total failure of device or system. Physical and electrical characteristics of PD mostly depends on diversity of PD occurrence and on material and characteristics of equipment [8]-[10].

\section{Classification of PD Detection Technique}

In the last 20-30 years research activities have been concentrated on developing methods of PD detection to identify, classify and locate PD in power equipment. By PD detection and fast response on occurred PD, diagnose of deterioration of insulation system and equipment will require less time [11]. Due this time many methods have been developed which categorized in four groups:

- Optical detection method

- Acoustic detection method

- Chemical detection method

- Electrical detection method

Categorization of PD detection is based on what emission of partial discharge they base the detection. For instance, optic detection is based on detection of light produced by PD, acoustic recording mechanical vibrations in material, chemical follows changes in chemical structure of materials caused by PD and lastly electrical methods focus on capturing electrical impulses from partial discharges [12]. PD phenomena must be located, measured, located and limited to insure quality of insulation system. Importance of PD detection lays in fact that it can help in maintenance of equipment, predict health and replacement time and operation efficiency of equipment, as well to help reduce and avoid losses caused by equipment failure. For better PD detection and diagnosis, sensors, processors and analyzers must be improved, with lowest possible cost. One of the important challenges in PD detection is elimination of field noises such as white Gaussian noise, frequency modulation (FM) signals and wireless communication signals [13]-[16].

\subsection{Optical Detection}

Optical detection of PD is based on recording light as result of ionization, excitation and recombination during discharge. Type of sensors that can be used in optical detection can be divided in three groups [17]:

- Imaging

- Quantitative non-imaging

- Optical or electrical detectors

In [18], optical detection method is introduced based on monitoring PD activities in dielectric test cell. Results showed this method has pros as sensitive and effective, compactible size, free of electromagnetic interference (EMI) and able to detect source and location of PD. In [17], optical detection is proposed in two groups which are surface discharge "outside" and surface discharge "inside" equipment. Detection of outside equipment is proposed to be done by DayCor camera and its operational diagram is presented as shown in Fig. 1.

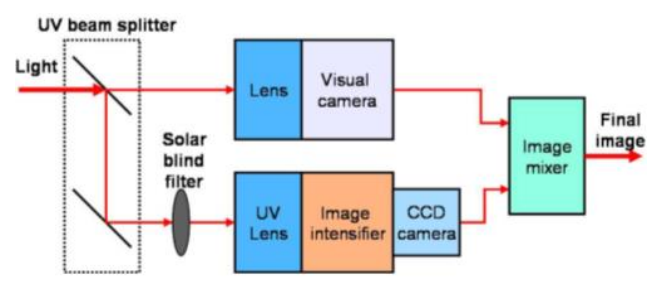

Fig. 1 - Operational diagram of DayCor Camera [17].

While detection "inside" of equipment is proposed to be based on optical that collects light and transmit it detection unit as shown in Fig. 2.

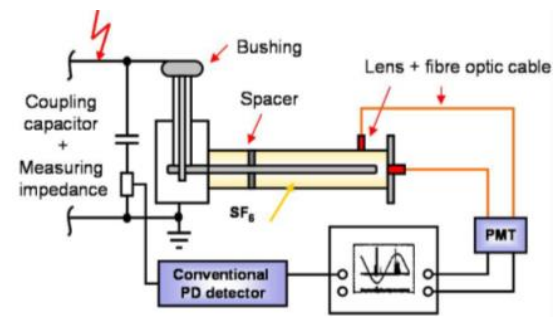

Fig. 2 - Experimental set up for partial detection inside equipment [17]. 
Optical detection has advantage over other methods since it can be used under impulse voltage conditions and it is not affected by environmental noise since PD and its detection happens in enclosed area [19].Files must be in MS Word only and should be formatted for direct printing, using the CRC MS Word provided. Figures and tables should be embedded and not supplied separately.

\subsection{Chemical Detection}

Phenomena of PD changes chemical characteristic of insulation which is used in developing methods of PD detection based on changes in chemical characteristics of insulation. The commonly methods are dissolved gas analysis (DGA) which provides information of PD in term of gas produce in insulation and high performance liquid chromatography (HPLC) is based on amount of by products produced in degradation of insulation such as glucose and forms of glucose [18].

DGA detection is based on volume and content of gas dissolved in insulation and in most cases presence of hydrogen is key indicator of PD signal. This method tends to be best and very effective of fault detection inside of transformer tank, with drawbacks that only can be used in off-line conditions and requires long time testing (as example air-circulation pump and gas chromatography). Some methods of on-line monitoring are hydrogen detection realized by placing fibre optic sensor and photoacoustic spectroscopy which highly sensitive method but without immunity to vibration and noise [18], [20].

In [21], hydrogen gas sensor and detection is presented. Sensor works on the way once it senses the hydrogen concentration, voltmeter will check change of resistance, as presented in Fig. 3. The implementation of sensor as shown in Fig. 4. The system consist of part for gas extraction built of gas chamber, vacuum chamber and gas sensing chamber. Detected concentration of gas will be sent to data acquisition system for analyzing level of PD signal. Another system presented in [21], is detection of gas produced by circulation of the air pump, presented in Fig. 5. One of the ways of hydrogen detection is based on Fiber Bragg grating (FBG) sensor [22]. This sensor is normally placed inside of optical fibre as shown in Fig. 6.

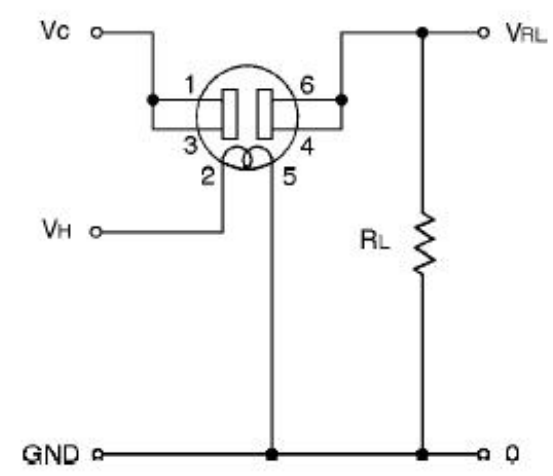

Fig. 3 - Hydrogen gas sensor measurement system [21].

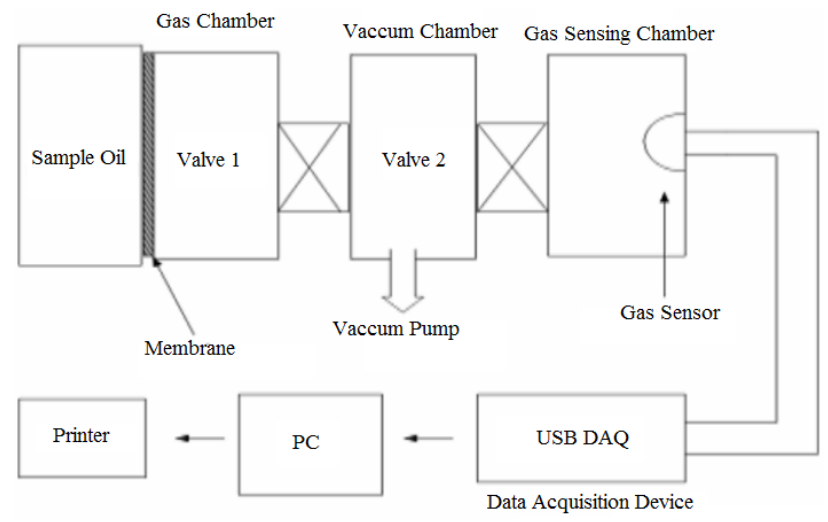

Fig. 4 - Hydrogen gas system detection with extraction part [21]. 


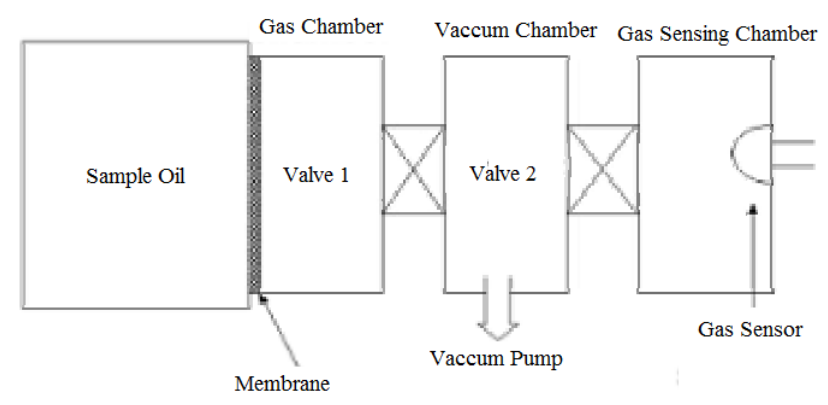

Fig. 5 - Gas detection of gas dissolved in air circulation [21].

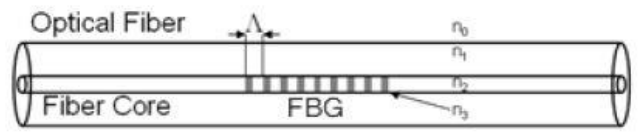

Fig. 6 - Fibre optic with FBG sensor [22].

The FBG sensor has thin palladium film inserted and physical changes initiated by hydrogen presence of this film. Immunity to EMI interferences, resistivity to high temperatures, strong dielectric strength, possibility of installation near to the insulation and point of interested are advantages of FBG sensor, leading to location of PD information in short and precise manner [22].

Chemical detection method has disadvantages due to cannot give information about source, strength and nature of partial discharge. In addition to this online monitoring using this method needs very complicated instruments and long analyzing processes [18].

\subsection{Acoustic Detection}

The aim of acoustic detection technique is sense and record the acoustic signal produced by PD activities [18]. PD is source of acoustic waves that goes through oil or insulating medium and can be detected on surface of tank or inside of the tank with suitable placed sensor [20]. PD in oil and insulation generally behaves as current stream, causing vaporization of insulation and as result mechanical explosion with acoustic emission is produced. To identify source it is necessary to capture signal produced by partial discharge that varies from $40 \mathrm{kHz}$ to $300 \mathrm{kHz}$ [12]. For better accuracy of PD detection, relationship between PD signal inside transformer, acoustic wave produced by PD and characteristics of wave spreading must be understood [19]. Acoustic signals can be detected by setting sensor at the surface of tank or inside of tank, while output can be analyzed by normal data acquisition systems [11]. Detection is done using wide selection of sensors such as microphones, piezoelectric, fibre sensors or accelerometers. In some cases combination of above is possible, as example optical fibre sensor acts as detector and reference point for piezoelectric sensor for location detection [20]. Results provided by these sensors and acquisition systems are based for common PD location techniques:

- Measurement of the time difference of arrival (TDOA) of the acoustic signal

- Measurement of the amplitude of acoustic signals in different areas of tank or standard auscultatory technique (SAT)

- Estimation of the direction of arrival (DOA) of acoustic signal

Piezoelectric (PZT) sensor is used in one of methods of acoustic detection PD, converting acoustic signal into electrical signal [20]. In order to choose suitable PZT sensor sensitivity must be considered to match bandwidth of acoustic signal. Sensor efficiency depends on acoustic impedance that matches with tested system. To ensure immunity of sensor on electromagnetic interferences sensor has insulating base available in different configurations.

PZT sensor is usually placed on external location of equipment like tank of transformer and works with ultrasonic frequencies as shown in Fig. 7. PZT sensor has some limitations due to oil to tank and tank to sensor interferences and signal received is attenuated, making elimination of noise hard and important. In most cases system are built in such manner that several sensors are used at once with characteristic of good locating of acoustic source based on time of arrival [24]. Example of such is presented in [23], with sensor placement presented in Fig. 8. 


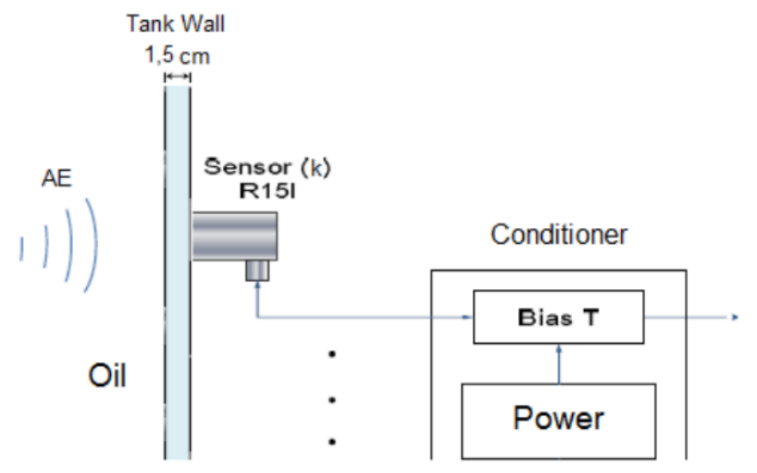

Fig. 7 - Example of acoustic PD detection with aid of PZT sensor [23].

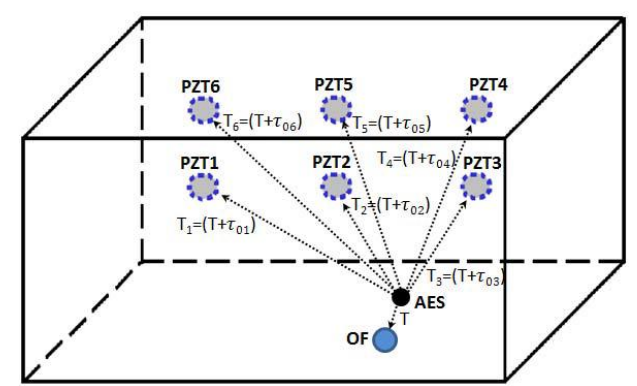

Fig. 8 - PD acoustic detection with system of several PZT sensors [23].

New methods such as optical fibre sensor have been developed and those kind of methods increase sensitivity, eliminating interfering signals with capability to survive in extreme conditions [25]. Once the fibre is inside the medium his location is more near to the source, where fibre has ability to detect stronger signal and since fibre is direct sensor it can cover all interior of tank [23].

Advantage of acoustic detection is that it is immune on EMI and it is possible to locate multiple source and location of PD in power equipment, such as oil-insulated transformers [18],[26]. This method is most applicable one and it is used in big range as tool in reliability centered maintenance, with very acceptable economic value. On other side problem with acoustic detection is in complex acoustic waveform and its spreading, waveform doesn't travel in perfect spherical form due to not homogeneous properties of equipment. Acoustic signal from PD goes through different mediums with different velocity and through its way can be interrupted and source of partial discharge is hard to detect [25].

\subsection{Electrical Detection}

Electrical detection has in interest capturing electrical and current pulses established in the void or impurity by streamer current, mostly used for detection of PD presence [18],[11]. By capturing current pulse level of charge can be measured and this level of charge will indicate certain conditions in insulation [20]. Electrical-ultrasonic method has ability to distinguish interference, very precise in localization, fast fading and has small range of detection [27]. Ultrasonic method can find location of PD, but it is not able to detect discharge quantity. On other side electrical PD detection has vice-versa characteristics, has ability to count number of discharge but can't locate source. Based on this electrical and ultrasonic are perfect match to cover each other's gaps [28]. Ultrahigh frequency or radio frequency (UHF RF) detection techniques method is very effective method eliminating interferences, with very high sensitivity and precise localization of PD. UHF RF method is non-contact and non-intrusive method suitable for any situation, thus it can classify type of PD occurred (surface, internal, corona) [29]. Installing of UHF sensors presents challenge since those sensor needs electromagnetic wave view into transformer as shown in Fig. 9. Some of options are window sensor on dielectric window on tank surface, probe sensors in oil valves or internal sensors inside of tank [30].

However electrical detection method has limitation since it is very hard to differentiate PD signal from external noise. Narrowband and broadband electrical noises during operation of high voltage equipment with short pulse width may lead to false identification of PD signal [18]. Distortion of PD signal on its way through equipment elements will lead to changes in characteristics of PD compared to characteristics on origin [18]. Electromagnetic interferences has a negative effects on sensitivity of electrical detection methods and because of this electrical detection gives mostly 
information about presence eliminating information about time and domain characterizes of partial discharge [11]. Sensitivity of electrical detection is related to capacitance of element being tested in such way that sensitivity decreases with capacitance increment. Location of PD is hard to determine using electrical detection method which is caused by complex structure of equipment such as transformers [11]. On-site and online application of electrical method would give less than accurate results, which limits this method only for off-line usage. The area where this method would be most applicable is routine testing of new equipment, then it is capable to provide valuable information on insulation conditions and can be used as parameter and benchmark for increasing capability of other methods [20].

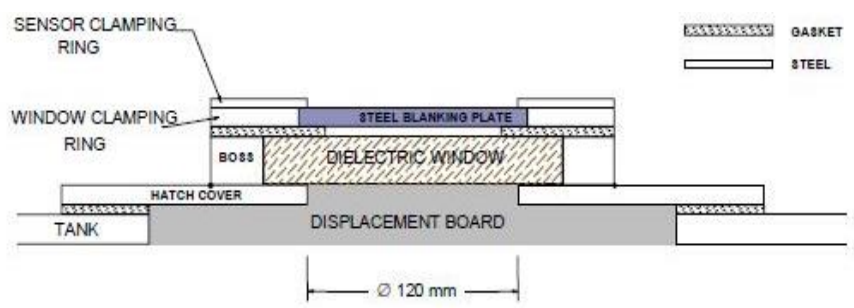

Fig. 9 - Dielectric window for UHF sensor [30].

\section{Conclusion}

According to the review study, monitoring and controlling of HV equipment is very important for reliable and efficient operation of such. Detection and analysis of PD topics has been discussed among researchers. Techniques of PD detection are grouped in electrical, acoustic, optical and chemical. They are differentiated on point of observation and each one has some disadvantages such as chemical detection is not able to applied online and on site, optical faces problem with transparency of equipment, electrical is not immune to EMI interferences and acoustic has problems with wave propagation through equipment. A lot of research has been done to overcome those disadvantages. In general, to develop ideal system for PD detection will take time until each method comes to perfection and by following trend of power equipment and devices. In most cases proposed solution to overcome gaps is combination of two or more techniques such as optical-acoustic method, electrical-acoustic method etc.

\section{References}

[1] Kunicki, M., Cichoń, A., and Nagi, L. Statistics based method for partial discharge identification in oil paper insulation systems. Electr. Power Syst. Res., (2018), pp. 1-13.

[2] Nafar, M., Niknam, T., and Gheisari, A., Using correlation coefficients for locating partial discharge in power transformer. Int. J. Electr. Power Energy Syst., Volume 33, No. 3, (2007), pp. 493-499.

[3] Hekmati, A., Proposed method of partial discharge allocation with acoustic emission sensors within power transformers. Appl. Acoust., Volume 100, (2015), pp. 26-33.

[4] Wang, Y. B. et al. Acoustic localization of partial discharge sources in power transformers using a particleswarm-optimization-route-searching algorithm. IEEE Trans. Dielectr. Electr. Insul., Volume 24, No. 6, (2017), pp. 3647-3656.

[5] Kunicki, M., Cichon, A., and Borucki, S. Measurements on partial discharge in on-site operating power transformer: A case study. IET Gener. Transm. Distrib., Volume 12, No. 10, (2016), pp. 2487-2495.

[6] Rohani, M. N. K. H., Yii, C.C., Isa, M., Hassan, S. I. S., Mukhtaruddin, A., and Yusof, N. A. Evaluation of Rogowski Coil Sensor Performance Using EMTP-ATP Software. Int. Conf. Electron. Des., (2016), pp. 446-451.

[7] Rohani, M. N. K. H. et al. Geometrical Shapes Impact on the Performance of ABS-Based Coreless Inductive Sensors for PD Measurement in HV Power Cables. IEEE Sens. J., Volume 16, no. 17, (2016), pp. 6625-6632.

[8] Mariprasath, T., and Kirubakaran, V. A real time study on condition monitoring of distribution transformer using thermal imager. Infrared Phys. Technol., Volume 90, (2018), pp. 78-86.

[9] Guillen, D., Idarraga-Ospina, G., and Mombello, E. Partial discharge location in power transformer windings using the wavelet Laplace function. Electr. Power Syst. Res., Volume 111, (2014), pp. 71-77.

[10] Liu, H. L. Acoustic partial discharge localization methodology in power transformers employing the quantum genetic algorithm. Appl. Acoust., Volume 102, (2016), pp. 71-78.

[11] Menon, R., Kolambekar, S., Buch, N. J., and Ramamoorty, M. Correlation of acoustic emission method and electrical method forlndetection of partial discharges in transformers. ICSD'01. Proc. 20001 IEEE 7th Int. Conf. Solid Dielectr. (Cat. No.01CH37117), no. i, (2001), pp. 299-302.

[12] Schwarz, R., Muhr, M., and Pack, S. Partial discharge detection in oil with optical methods. IEEE Int. Conf. Dielectr. Liq. 2005. ICDL 2005. (2005), pp. 245-248. 
[13] Yii, C. C., Rohani, M. N. K. H., Isa, M., and Hassan, S. I .S. Multi-end PD location algorithm using segmented correlation and trimmed mean data filtering techniques for MV Underground Cable. IEEE Trans. Dielectr. Electr. Insul., Volume 24, No. 1, (2017), pp. 92-98.

[14] Cunha, C. F. F. C., Carvalho, A. T., Petraglia, M. R., and Lima, A. C. S. A new wavelet selection method for partial discharge denoising. Electr. Power Syst. Res., Volume 125, (2015), pp. 184-195.

[15] Allahbakhshi, M., and Akbari, A. A method for discriminating original pulses in online partial discharge measurement. Meas. J. Int. Meas. Confed., Volume 44, No. 1, (2011), pp. 148-158.

[16] Mota, H. D. O., Da Rocha, L. C. D., Salles, T. C. D. M., and Vasconcelos, F. H. Partial discharge signal denoising with spatially adaptive wavelet thresholding and support vector machines. Electr. Power Syst. Res., Volume 81, No. 2, (2011), pp. 644-659.

[17] Schwarz, R., and Muhr, M. Modern technologies in optical partial discharge detection. Conf. Electr. Insul. Dielectr. Phenom., (2007), pp. 163-166.

[18] Karmakar, S., Roy, N. K., and Kumbhakar, P. Detection of partial discharges in a high voltage equipment. J. Electr. Eng., (2006), pp. 1-6.

[19] Kaufhold, M., Bamji, S. S., and Bulinski, A. T. Optical detection of partial discharges in gas-insulated systems. Conf. Electr. Insul. Dielectr. Phenom. (CEIDP), Annu. Rep., Volume 2, (1996), pp. 618-623.

[20] Mukhtaruddin, A., Isa, M., Adzman, M. R., Hasan, S. I. S., Rohani, M. N. K. H., and Yii, C. C. Techniques on partial discharge detection and location determination in power transformer. Int. Conf. Electron. Des., (2016), pp. 537-542.

[21] Seo, H.-J. S. H.-J., Hwang, K.-H. H. K.-H., and Rhie, D.-H. R. D.-H. Development of gas detector for hydrogen dissolved in transformer oil. IEEE Instrum. Meas. Technol. Conf. Proc. 2005, Volume 1, (2005), pp. 17-19.

[22] Ma, G., Li, C., Mu, R., and Jiang, J., Fiber Bragg grating sensor for hydrogen detection in power transformers. IEEE Trans. Dielectr. Electr. Insul., Volume 21, No. 1, (2014), pp. 380-385.

[23] Búa-núñez, I., Posada-román, J. E., Rubio-serrano, J., and Garcia-souto, J. A. Instrumentation system for location of partial discharges using acoustic detection with piezoelectric transducers and optical fiber sensors. IEEE Trans. Instrum. Meas., Volume 63, No. 5, (2014), pp. 1002-1013.

[24] Sikorski, W., Siodla, K., Moranda, H., and Ziomek, W. Location of partial discharge sources in power transformers based on advanced auscultatory technique. IEEE Trans. Dielectr. Electr. Insul., Volume 19, No. 6, (2012), pp. 1948-1956.

[25] Kim, T. Y., Suh, K. S., Nam, J. H., and Takada, T. Acoustic monitoring of HV equipment with optical fiber sensors. IEEE Trans. Dielectr. Electr. Insul., Volume 10, No. 2, (2003), pp. 266-270.

[26] Zhang, W., Chen, Q., and Zhao, H. Numerical investigation of acoustic emissions distribution from partial discharge in transformer. Int. Conf. Electr. Mater. Power Equip., (2017), pp. 535-538.

[27] Miao, P., Li, X., Yue, H., Sheng, G., Ye, H., and Jiang, X. Acoustic-electrical based detection system for partial discharge localization of GIS. Proc. - Power Eng. Autom. Conf. PEAM 2012, (2012), pp. 1-5.

[28] Jiang, W., Liu, F., Wang, Z., and Zhang, X. Transformer partial discharge detection using electrical-ultrasonic technology. Asia-Pacific Power Energy Eng. Conf., (2012), pp. 2-5.

[29] Giussani, R., Cotton, I., and Sloan, R. Comparison of IEC 60270 and RF partial discharge detection in an electromagnetic noise-free environment at differing pressures. IEEE Int. Symp. Electr. Insul., (2012), pp. 127131.

[30] Judd, M. D. Experience with UHF partial discharge detection and location in power transformers. Electr. Insul. Conf., (2011), pp. 201-205.Van der Geer, J., Hanraads, J. A. J., \& Lupton, R. A. (2000). The art of writing a scientific article. Journal of Science Communication, 163, 51-59. 